\title{
New Concept of Ocular Implications in COVID-19 Infection: A Brief Review
}

\author{
Apen Hoddor Silaban
}

\section{ABSTRACT}

Coronavirus disease 2019 is a novel pneumonia-like respiratory illness initiated by the infection of the severe-acute-respiratory-syndrome coronavirus 2 (SARS-CoV-2). This type of virus invades the human body through various intermediaries, including through the eye. The presence of a coronavirus invasion in the eye may cause several ocular manifestations, which can be an initial clinical finding of a coronavirus infection in the host body. On the other hand, it can also cause systemic complications that may affect the eyes. This review will discuss in more detail how the coronavirus can infect humans through the eye, explain its manifestations, and briefly explain the proper and prompt management steps that must be taken.

Keywords: Coronavirus, eye, infection.

Published Online: January 7, 2021

ISSN: $2593-8339$

DOI: $10.24018 /$ ejmed.2021.3.1.630

\section{A. H. Silaban*}

Lende Moripa Christian Hospital, West Sumba, East Nusa Tenggara, Indonesia.

(e-mail: apensilabandr ${ }^{\circledR}$ gmail.com)

*Corresponding Author

\section{INTRODUCTION}

Human infections caused by the severe acute respiratory syndrome coronavirus 2 (SARS-CoV-2) were originally reported in late 2019, which is called the coronavirus disease 19 (COVID-19) [1]. This disease is spreading rapidly worldwide, as the World Health Organization (WHO) has declared the disease as a new pandemic in March 2020 [2]. Almost a year since the first known case in the Huanan wholesale sea market in Wuhan, China, it has been associated with over 1 million mortalities worldwide, which is accountable for $55 \%$ of the total cases [3]. Ioannidis and colleagues reported that nearly $10 \%$ of the worldwide population might have been infected by November 2020 . The case fatality rate is about $0.15-0.20 \%$ in those people under 70 years [4]. COVID-19 is transmitted by respiratory droplets from one person to another, mainly by close contact. The ocular surface is one of the most exposed parts of the body to the external environment; thus, it suits as a potential transmission gateway for any pathogens, such as coronaviruses, to infect the human body [5].

COVID-19 has been previously stated to be correlated with viral conjunctivitis in humans. Additionally, experimental studies reported from animal models infected by SARS-CoV2 to the retina showed to cause retinal vasculitis and retinal degeneration [6]. Contrary, conjunctivitis also seems to be an atypical clinical finding among patients with confirmed diagnosis of COVID-19, as it has only been reported in less than $1 \%$ of the general cases in China [7].

A recent case series in Hubei province, China, found several ocular manifestations from $12(31.6 \%)$ of 38 hospitalized COVID-19 patients, including three ocular hyperemia cases, seven cases of chemosis, and seven cases of epiphora [8]. However, ophthalmic presentations and complications of COVID-19 infection have not been widely discussed [9]. This review aims to identify and analyze the ocular manifestation of COVID-19 infection to help clinicians build a prompt diagnosis and management regarding COVID-19 infection to the eye.

\section{Coronavirus Diseases 2019 (COVID-19)}

Coronavirus is categorized as an enveloped, positive single-strand ribonucleic acid (RNA) virus. It is the part of the Orthocoronavirinae subfamily, as the name proposes that the group consists of viruses that have a characteristic of a "crown-like" spike on their surface [10]. The diameter of SARS-CoV-2 is $80-120 \mathrm{~nm}$. There are four known types of the virus, including the $\alpha$-coronavirus, $\beta$-coronavirus, $\gamma$ coronavirus, and $\delta$-coronavirus [11]. Transmission occurs mainly through respiratory droplets and aerosols from both asymptomatic or symptomatic infected individuals [12], [13]. The most common transmission method by droplets is emitted during talking, coughing, or sneezing, which later reach the mucosal surfaces, including the mouth, nose, and eyes. The incubation period is up to 2 to 14 days, and the infectivity usually causes mild to moderate severity. Fever is the most common systemic symptom, followed by dry cough and fatigue [14].

\section{ANTERIOR OCULAR MANIFESTATIONS OF COVID-19}

The COVID-19 may invade the eyes, even if only a few reports are available on ocular disorders that occurred during the COVID-19 infection. Anterior ocular manifestations such as conjunctivitis and anterior uveitis are the most frequent 
ocular manifestation currently reported in the literature [15]. A study by $\mathrm{Wu}$ and colleagues presented the findings of 38 COVID-19 cases in Hubei Province, China, with $31.6 \%$ of total cases demonstrated ocular manifestations consistent with conjunctival hyperemia, chemosis, epiphora, and ocular discharges. There was only a single case presented with conjunctivitis as an initial clinical symptom [16]. A similar report also suggests that COVID-19 may develop conjunctivitis, both as an initial sign of infection or during hospitalization in severe COVID-19 illness [15].

Loffredo and colleagues conducted a meta-analysis presenting an overall rate of conjunctivitis cases in COVID19 patients was $1.1 \%$, divided into $3 \%$ in severe cases and $0.7 \%$ in mild-to-moderate cases. The study also summarized that conjunctivitis is more common in severe COVID-19 and may become a warning sign of an unsatisfying prognosis [17]. A prospective study by Hong and colleagues reported that $15(27 \%)$ of 56 confirmed COVID-19 cases had aggravated ocular manifestations. About six patients (11\%) had prodromal ocular manifestation earlier than the onset of systemic COVID-19 manifestation. They also summarized that COVID-19 ocular manifestations are quite common and usually occur early after infection [18]. Another metaanalysis by Aggarwal and colleagues noticed that from 2,347 COVID-19 patients, the most common ocular presentations were eye-aching (31.2\%), ocular discharges (19.2\%), conjunctivitis $(10.8 \%)$, followed by follicular conjunctivitis (7.7\%) [19].

Other cases reported by Cheema and colleagues found keratoconjunctivitis as the main finding, with symptoms of conjunctival hyperemia, photophobia, and watery discharge [20]-[23]. Ophthalmic findings are usually indistinguishable from mild follicular conjunctivitis (Fig. 1), such as eyelid edema, conjunctival congestion with/without pseudomembrane, and follicular reaction of palpebral [21]-[23]. The patient may also have pseudo-dendrites (Fig. 2), which perhaps come from healed epithelial defects or progressing subepithelial infiltrate. However, the onset of the appearance of COVID-19 on the epithelium cells of conjunctiva and tears are still unclear; thus, more studies are required to evaluate the transmissibility of the virus by ocular gateway in asymptomatic COVID-19 patients [21].

A retrospective study from $\mathrm{Lu}$ and colleagues tested conjunctival samples of the virus RNA in 30 confirmed COVID-19 cases. The finding of positive viral RNA on the conjunctiva appeared three days after the onset [24]. From such a finding, it can be concluded that the SARS-CoV-2 may enter and cause anterior ocular manifestations in the eye. The pathogenesis behind this finding is started when the virus attached to angiotensin-converting enzyme 2 (ACE-2), known as the receptor for SARS-CoV-2 in the eye (Fig. 3) [25]-[27].

Based on all the findings above, it can be concluded that the clinicians should be more aware and ensure that a comprehensive review of clinical respiratory symptoms, transmittable contact, and travel history is completely carried out during the interview, especially when facing patients presented with conjunctivitis. That is because the patients who have conjunctivitis accompanied by shortness of breath and cough; and previously traveled to a place with an acknowledged status of COVID-19 outbreaks are at greater risk of having this deadly infection [28].

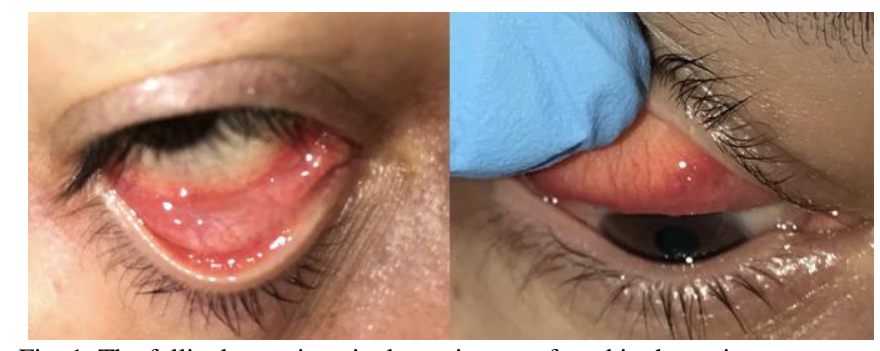

Fig. 1. The follicular conjunctival reaction was found in the patient two days before the confirmation of COVID-19 [20].

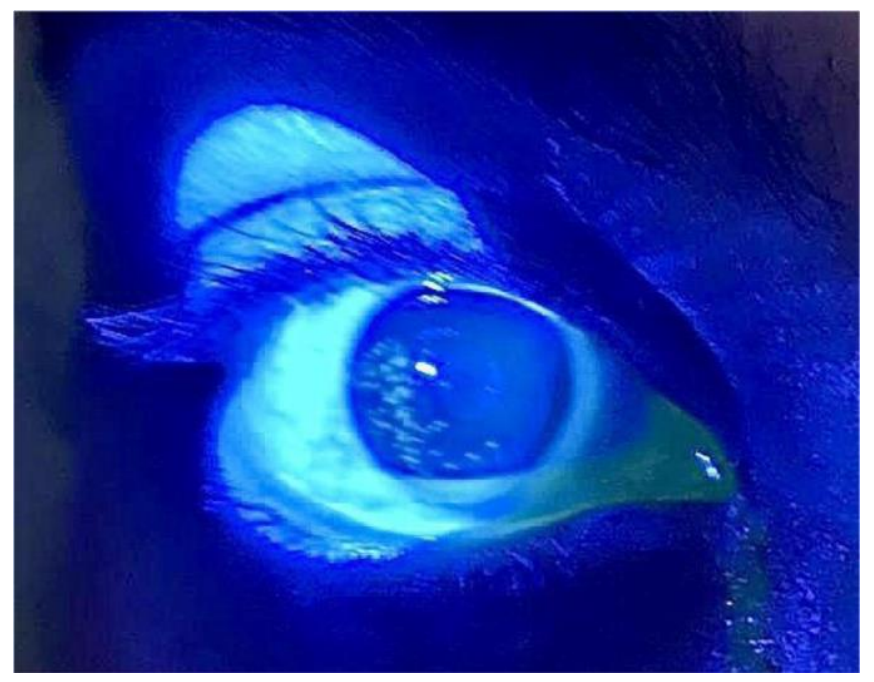

Fig. 2. External photograph using fluorescein eye photographed under cobalt blue light with epithelial changes found in COVID-19 case [23].

\section{POSTERIOR OCULAR MANIFESTATIONS OF COVID-19}

Only a few reports are available on posterior ocular disorders during COVID-19 infection, with retinitis and optic neuritis being the most frequent ocular manifestation [15]. The latest case report proposed changes of retinal structure related to COVID-19 infections. Eleven cases examined under Optical Coherence Tomography (OCT) showed hyperreflective lesions at the region of retinal layers, including the inner plexiform layer and ganglion cells layer, flagrantly at the papillomacular bundle. However, neither ocular disturbances nor the changes in ganglion cell complex analysis and OCT angiography are related to this finding. There were four cases with subtle cotton wool spots and microhemorrhages along the retinal findings [29].

Invernizzi and colleagues reported retinal disorders in several cases with confirmed COVID-19 (Fig. 4). They also stated that it was difficult to determine whether retinal ablation and cotton wool spots occurred secondary to COVID-19 infection or merely incidental findings, knowing that the patients presented with a prolonged history of hypertension and diabetes. However, the authors did not find any relationship between the systemic comorbidities and the retinal dysfunctions. The findings are possibly due to the inflammatory response related to SARS-CoV-2 itself; or related to the medication, which may disturb the fragile microvasculature aggravated by the underlying conditions [29]. Dal Negro and colleagues suggested that the dilation of venous vasculature and retinal hemorrhage may be caused by venous thrombotic, hypercoagulability, and dysregulation of 
immune response likely represent the main pathogenetic determinants of severe ocular complication [30].

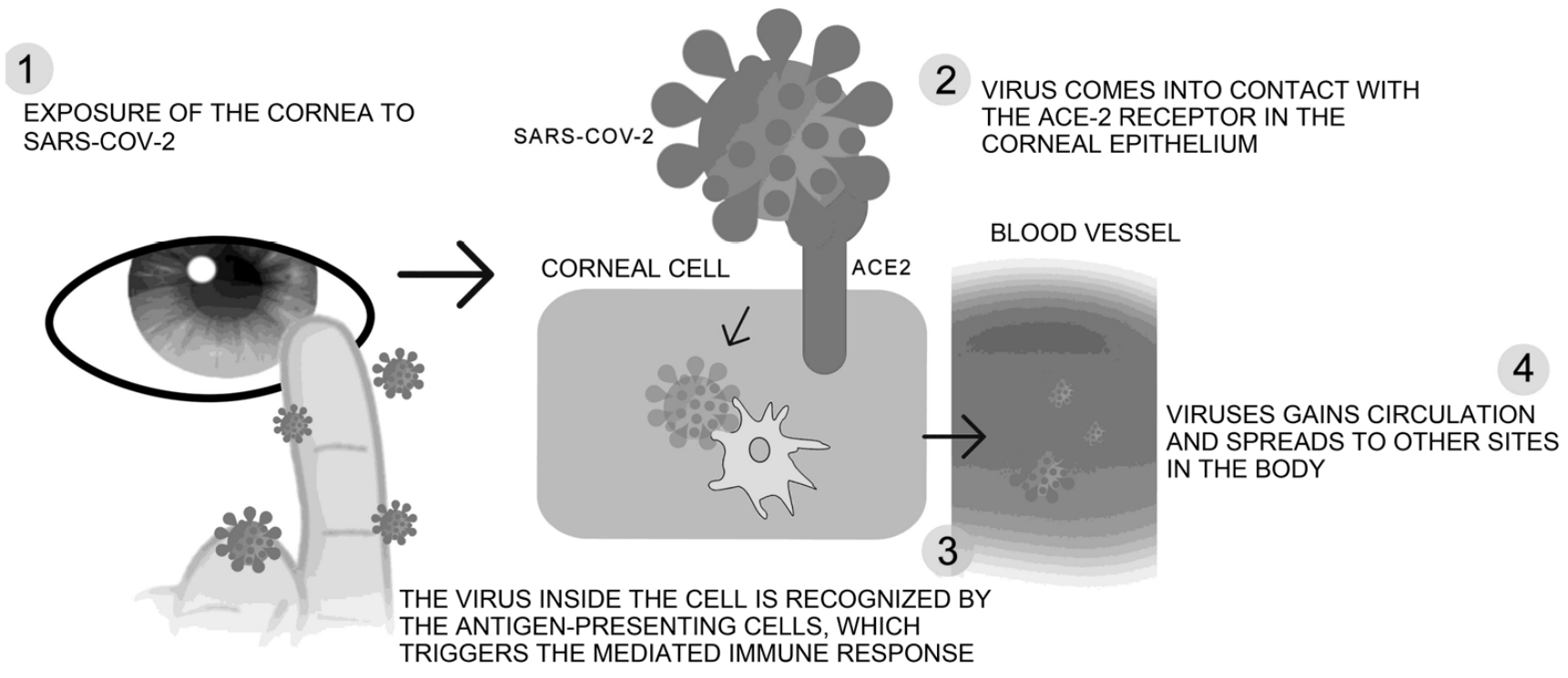

Fig.3. Penetration of SARS-CoV-2 to the conjunctival surface [27].
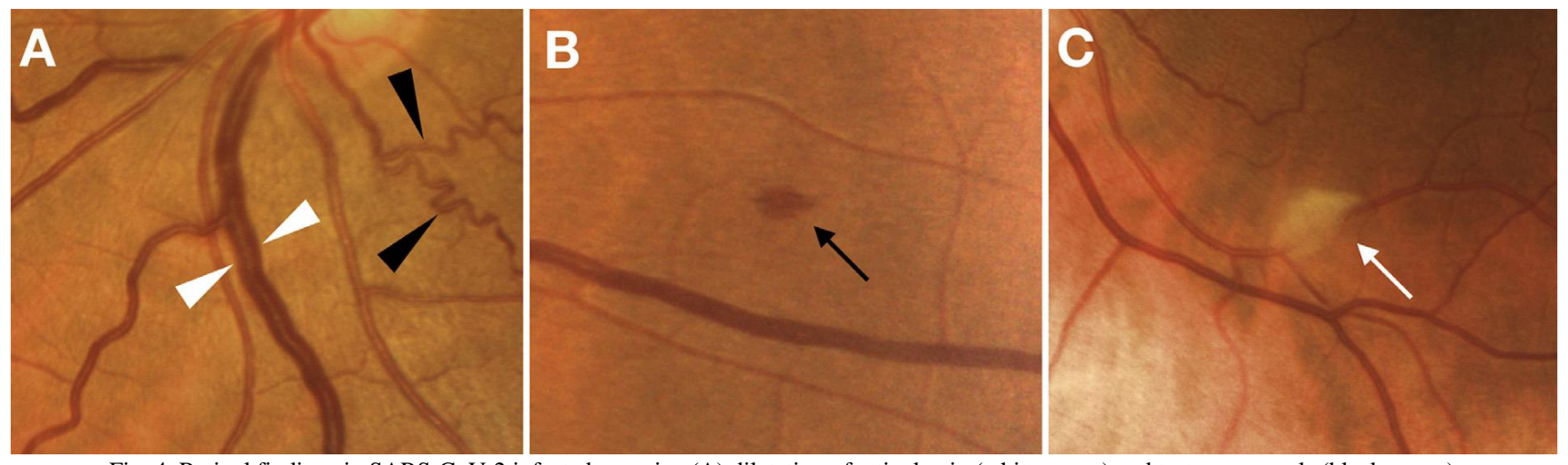

Fig. 4. Retinal findings in SARS-CoV-2 infected eyes, i.e. (A) dilatation of retinal vein (white arrow) and tortuous vessels (black arrow),

(B) retinal hemorrhage, and (C) appearance of cotton wool spot [29].

A study reported that SARS-CoV-2 might cause choroiditis with retinal detachment, granulomatous anterior uveitis, and retinal vasculitis [15]. Results from blood tests indicated a higher count of white blood cells and neutrophils, C-reactive protein, lactate dehydrogenase, and procalcitonin compared to patients COVID-19 with normal ocular findings [8]. Additional examination using a biomicroscopic slit lamp should be considered, especially with the non-contact technique. It is also better if the doctor and patient do not communicate with each other to prevent the transmission of the virus during the close examination.

\section{MEChANiSM OF TRANSMISSION}

Nasolacrimal duct is a canal that mediates the ocular surface and the upper respiratory tract. Any fluid particle dropped on the ocular surface is partially absorbed into the conjunctiva. Still, any excess amount will be drained into the nasal cavity through the nasolacrimal duct and then transferred towards the nasopharynx and trachea. This condition will allow any pathogens exposed on the ocular surface to be transferred to respiratory mucosa. Moreover, recent studies have reported that ophthalmic and respiratory mucosa have the same respiratory pathogen receptor [31].
One of which is the ACE-2 receptor that is highly found on alveolar epithelial cells, the small intestine, and the kidney (Fig. 2). ACE-2 is known as a type-I transmembrane enzyme [32]. SARS-CoV-2 invades the body through ACE-2 as a cellular entry point to the receptor [33].

The expression of ACE-2 is also found in the epithelium of the conjunctival or cornea. However, this ocular expression is considerably lesser than in other tissues [27]. The capacity of binding between the ACE-2 protein on conjunctival cells and the SARS-CoV-2 spike protein is much lower than that in the lung tissue [34]. The capabilities of the virus to invade the host cells depend on (1) the penetration of the virus, (2) the number of receptors on the host cell membrane to bind the virus, and (3) the host immune status. The virus attaching to the host cell membrane by ACE- 2 receptors is recognized as the primary step and the most crucial part for the SARS-CoV2 invasion [35].

\section{DETECTION OF COVID-19 AT THE OCULAR SURFACE}

The presence of COVID-19 in the eye is known as the result of (1) direct exposure of the virus from aerosol particles or droplets to the conjunctiva; (2) through the migration of the virus from the upper respiratory tract; or (3) exudation 
through vascularization of infected conjunctiva.

Conjunctival swab was carried out in a case of COVID-19 with keratoconjunctivitis and became helpful to confirm the presence of SARS-CoV-2 RNA on the conjunctival surface on the second day of the onset [35]. This examination was also done in a patient who complained of bilateral conjunctivitis as the initial symptom and found the virus RNA on the $21^{\text {st }}$ day of the onset [36]. Conjunctival swab was also done in many studies, including among the COVID-19 patients with no ocular symptoms. Sixty-four tear samples were taken using the Schirmer strip from a total of 17 COVID-19 patients. The samples were adopted during the $21^{\text {st }}$ day of the onset and then analyzed using PCR to discover the presence of virus RNA [15]. Although a positive presence of virus was detected on nasopharyngeal swabs, the authors did not find any viral RNA from the tear samples [15]. Zhong and colleagues predicted that the sensitivity value of COVID19 detection from conjunctival sac is merely up to $7 \%$, which is relatively low, so that it becomes unreliable to examine viral load on the ocular surface. Currently, there has not been any particular standard of conjunctival SARS-CoV-2 detection for the diagnosis of COVID-19 [37].

A prospective study involving 17 COVID-19 patients in Singapore underwent serial nasopharyngeal swabs and tears sampling using Schirmer strip. The results of all the samples were negative. However, another study investigating the viral RNA on the conjunctival surface from asymptomatic ocular manifestations showed that three out of 67 cases had positive results [38]. The mentioned studies confirm that SARS-CoV2 can exist in the conjunctival sac, but that transmission of SARS-CoV-2 through the ocular gateway remains unclear. Nevertheless, the small number of positive results might also be confounded by the examination methods and conditions [39].

Several possibilities might be accountable for the low rate of viral RNA in conjunctival sacs and tears, such as the sampling time, viral concentration, and examination approach, which may also affect the sensitivity rate [40]. The samples could be taken at certain times where the presence of viral RNA was not at its highest level, which is generally at the early onset of the disease. On the other hand, the collection technique is rather diverse, including the variety of swabs. The calcium alginate content in the swabs may inactivate the virus, as well as the use of topical anesthesia during the swab collection may also affect the viability of the virus [41]. Notably, many studies also reported that patients with COVID-19 often require repetition of nasopharyngeal swabs at different times to confirm the result. More studies should consider the relationship between viral load and viral shedding in ocular systems.

\section{Management of OCUlaR Manifestations IN COVID-19}

Chloroquine $(2.3 \mathrm{mg} / \mathrm{kg})$ and its derivates, including hydroxychloroquine (less than $5.0 \mathrm{mg} / \mathrm{kg}$ ) once a day, has both anti-inflammation and antiviral properties that are known as potential treatment for patient with ocular manifestations caused by COVID-19 [42]. Nonetheless, some studies suggest that additional evidences from multicenter trials are still required, despite the favorable outcomes reported from the use of this preparation. Retinopathy caused by the use of hydroxychloroquine is infrequently seen before ten years of the treatment at the recommended dose [43]. Nevertheless, the recommended doses needed in the treatment of COVID-19 infection are four times higher; thus, the potential side-effects and complications should be carefully monitored during the therapy. Some studies recommended to carry out the fundoscopy assessment before, throughout, and subsequently to the treatment. Some ophthalmologists have also suggested lopinavir/ritonavir preparation with the recommended dose of 400/100 mg twice a day, and the treatment duration up to seven days. Sideeffects, such as retinal toxicity, can be found, although considered unlikely among COVID-19 patients [44].

The American Academy of Ophthalmology (AAO) suggested that all clinicians deliver only urgent or emergent care to lessen any possible contact of SARS-CoV-2 transmission. It is also essential to frequently changes disposable medical instruments. In the presence of eye problems without any significant eye pain, vision disturbances, or unusual light oversensitivity, it is best to limit the medication use and consider applying cold eye compression, artificial tears, or lubricating ophthalmic ointment. A short-term application of topical antibiotics combination with lubricant, i.e., moxifloxacin hydrochloride $0.5 \%$ or ciprofloxacin hydrochloride $0.3 \%, 4-6$ times a day for 14 days, followed by sodium hyaluronate $0.1 \%$ or carboxymethyl cellulose $0.5 \%, 4-6$ times a day for 14 days can be prescribed to prevent potential bacterial infection among contact lens wearer [45], [46].

Analgesia agents containing non-steroidal antiinflammatory drugs (NSAIDs), i.e., ibuprofen, should not be used as it may worsen the systemic effects of COVID-19 [47]. In case of pain, oral preparation of paracetamol can be considered. Topical steroids should not be used due to the possible escalation of intraocular pressure. Ophthalmologists may consider the use of chloroquine eye drops $0.03 \%$, two times a day [48]. Additionally, a rare case of bilateral pseudomembranous conjunctivitis in an intubated COVID-19 patient possessed a good outcome treated by azithromycin eye drops, two times a day, combined with low doses of dexamethasone and mechanical debridement [49].

\section{CONCLUSION}

SARS-CoV-2 is a virus responsible for this pandemic. It affects the eye and may present signs of ocular manifestation. Ocular transmission by exposure to conjunctival surface is possible; thus, the eye may serve as a portal of penetration for the SARS-CoV-2, leading to systemic infection. Clinicians should be the first providers to assess all the patients conceivably infected with COVID-19 as patients may present with conjunctivitis. Prevention such as eye protection by face shields and goggles are essential to prevent viral transmission, especially among clinicians. Diagnosis and management in COVID-19 patients presented with ocular symptoms must be made by early screening or nasopharyngeal swabs to exclude the co-infection in this pandemic era. 


\section{REFERENCES}

[1] World Health Organization. (August 2020). Coronavirus Disease (COVID-2019) Situation Report [Online]. Available: https://www.who .int/emergencies/diseases/novel-coronavirus-2019/situationreports/

[2] World Health Organization. (February 2020). Report of the WHOChina Joint Mission on Coronavirus Disease 2019 (COVID-19) [Online]. Available: https://www.who.int/publications/i/item/report-of -the-who-china-joint-mission-on-coronavirus-disease-2019-(covid19)

[3] Pan American Health Organization/World Health Organization. (October 2020). Epidemiological alert recurring waves and outbreaks of COVID-19 [Online]. Available: https://www.paho.org/en/docu ments/epidemiological-alert-recurring-waves-and-outbreaks-covid-19

[4] J.P.A. Ioannidis, "Global perspective of COVID-19 epidemiology for a full-cycle," Eur J Clin Invest., vol. 50, pp. 1-9, 2020.

[5] X. Xu, P. Chen, J. Wang, et al., "Evolution of the novel coronavirus from the ongoing Wuhan outbreak and modeling of its spike protein for risk of human transmission," Sci China Life Sci., vol. 63, no. 3, pp. 457 460, 2020.

[6] Y. Wang, B. Detrick, Z.X. Yu, et al., "The role of apoptosis within the retina of coronavirus-infected mice," Invest Ophthalmol Vis Sci., vol. 41, pp. 3011-3018, 2000.

[7] W.J. Guan, Z.Y. Ni, Y. Hu, et al., "China medical treatment expert group for Covid-19: clinical characteristics of coronavirus disease 2019 in China," N Engl J Med., vol. 382, pp. 1708-1720, 2020.

[8] J.O. Li, D.S.C. Lam, Y. Chen, et al., "Novel coronavirus disease 2019 (COVID-19): The importance of recognising possible early ocular manifestation and using protective eyewear," The British Journal of Ophthalmology, vol. 104, no. 3, pp. 297-298, 2020.

[9] J. Xia, J. Tong, M. Liu, et al., "Evaluation of coronavirus in tears and conjunctival secretions of patients with SARS-CoV-2 infection," J Med Virol., vol. 92, no. 6, pp. 589-594, 2020.

[10] S. Perlman, "Another decade, another coronavirus," N Engl J Med., vol. 382, pp. 760-762, 2020.

[11] W. Wang, J. Tang, F. Wei, "Updated understanding of the outbreak of 2019 novel coronavirus (2019-nCoV) in Wuhan, China," J Med Virol., vol. 92 , no. 4, pp. 441-447, 2020.

[12] C. Rothe, M. Schunk, P. Sothmann, et al., "Transmission of 2019nCoV infection from an asymptomatic contact in Germany," $N$ Engl J Med., vol. 382, no. 10, pp. 970-971, 2020.

[13] A.M. Abdullahi, "Virology and epidemiology of the 2019 coronavirus disease (COVID-19)," J Commn Med Pub Health Rep., vol. 1, no. 1, pp. 1-9, 2020.

[14] T.H. Lai, E.W. Tang, S.K. Chau, et al., "Stepping up infection control measures in ophthalmology during the novel coronavirus outbreak: an experience from Hong Kong," Graefe's Archive for Clinical and Experimental Ophthalmology, pp. 1-7, 2020.

[15] I. Seah, R. Agrawal, "Can the coronavirus disease 2019 (COVID-19) affect the eyes? A review of coronaviruses and ocular implications in humans and animals," Ocul Immunol Inflamm., vol. 28, no. 3, pp. 391$395,2020$.

[16] P. Wu, F. Duan, C. Luo, et al., "Characteristics of ocular finding of patients with coronavirus disease 2019 (COVID-19) in Hubei Province, China," JAMA Ophthalmology, vol. 138, no. 5, pp. 575-578, 2020.

[17] L. Loffredo, F. Pacella, E. Pacella, et al., "Conjunctivitis and COVID19: a meta-analysis," J Med Virol., pp. 1-2, 2020.

[18] N. Hong, W. Yu, J. Xia, et al., "Evaluation of ocular symptoms and tropism of SARS-CoV-2 in patients confirmed with COVID-19," Acto Ophthalmol., pp. 1-7, 2020.

[19] K. Aggarwal, A. Agarwal, N. Jaiswal, et al., "Ocular surface manifestations of coronavirus disease 2019 (COVID-19): a systematic review and meta-analysis," PLoS ONE, vol. 15, no. 11, pp. 1-20, 2020.

[20] Z.K. Ozturker, "Conjunctivitis as sole symptom of COVID-19: a case report and review of literature," EJO, pp. 1-6, 2020.

[21] K. Hu, J. Patel, B.C. Patel. January 2020. Ophthalmic manifestations of coronavirus (COVID-19). StatPearls (Online). Available https:// www.ncbi.nlm.nih.gov/books/NBK556093/

[22] V. Navel, F. Chiambaretta, F. Dutheil, "Haemorrhagic conjunctivitis with pseudomembranous related to SARS-CoV-2," Am J Ophthalmol Case Rep., vol. 19, pp. 1-3, 2020.

[23] M. Cheema, H. Aghazadeh, S. Nazarali, et al., "Keratoconjunctivitis as the initial medical presentation of the novel coronavirus disease 2019 (COVID-19)," Can J Ophthalmol., vol. 55, no. 4, pp. e125-e129, 2020.

[24] C.W. Lu, X.F. Liu, Z.F. Jia, "2019-nCoV transmission through the ocular surface must not be ignored," Lancet, vol. 395, no. 10224, pp. e39, 2020
[25] P. Senanayake, J. Drazba, K. Shadrach, et al., "Angiotensin II and its receptor subtypes in the human retina," Invest Ophthalmol Vis Sci., vol. 48, no. 7, pp. 3301-3311, 2007

[26] S.L.L. Yan, X. Pan, M. Jing, "Mechanism of the action between the SARS-CoV S240 protein and the ACE2 receptor in eyes," J Virol., vol. 6, pp. 783-786, 2006.

[27] B.R.S. Torres, C.E.X. da Cunha, L.R. Castro, et al., "Ocular manifestations of COVID-19: a literature review," Rev Assoc Med Bras., vol. 66, no. 9, pp. 1296-1300, 2020.

[28] American Academy of Ophthalmology. March 2020. Alert: important coronavirus updates for ophthalmologists (Online). Available: www. aao.org/headline/alert-important-coronavirus-context.

[29] A. Invernizzi, A. Torre, S. Paruulli, et al., "Retinal findings in patients with COVID-19: result from the SERPICO-19 study," EClinical Medicine, vol. 27, pp. 1-9, 2020.

[30] R.W. Dal Negro, P. Turco, G. Santin, "Ocular venous thrombosis as the main manifestation of an otherwise pauci-symptomatic COVID19," Clin Case Rep Int., vol. 4, pp. 1-4, 2020.

[31] I. Hammin, W. Timens, M.L.C. Bulthuis, et al., "Tissue distribution of ACE2 protein, the functional receptor for SARS coronavirus: a first step in understanding SARS pathogenesis," J Pathol., vol. 203, no. 2, pp. 631-637, 2020.

[32] J.F. Riordan, "Angiotensin-I-converting enzyme and its relatives," Genome Biology, pp. 225, 2003.

[33] M. Hoffman, H. Kleine-Weber, S. Schroeder, et al., "SARS-CoV-2 cell entry depends on ACE2 and TMPRSS2 and is blocked by a clinically proven protease inhibitor," Cell, vol.181, no. 2, pp. 271-278, 2020.

[34] Y. Sun, L. Liu, X. Pan, et al., "Mechanism of the action between the SARS-CoV S240 protein and the ACE2 receptor in eyes," Int $J$ Ophthalmol., vol. 6, no. 4, pp. 783-786, 2006

[35] A. Milewska, P. Nowak, K. Owczarek, et al., "Entry of human coronavirus NL63 into the cell," J Virol., vol. 92, no. 3, pp. e01933-17, 2018.

[36] F. Colavita, D. Lapa, F. Carletti, et al., "SARS-CoV-2 isolation from ocular secretions of a patient with COVID-19 in Italy with prolonged viral RNA detection," Ann Intern Med., pp. 1-3, 2020.

[37] Y. Zhong, K. Wang, Y. Zhu, et al., "COVID-19 and the eye”, Journal of Infection, pp. 122-123, 2020.

[38] Y. Zhou, Y. Zeng, Y. Tong, et al., "Ophthalmologic evidence against the interpersonal transmission of 2019 novel coronavirus through conjunctiva," MedRxiv, pp. 1-16, 2020.

[39] X. Zhang, X. Chen, L. Chen, et al., "The infection evidence of SARSCOV-2 in ocular surface: a single-center cross-sectional study," MedRxiv, pp. 1-12, 2020

[40] Z.A. Memish, J.A. Al-Tawfiq, H.Q. Makhdoom, et al., "Respiratory tract samples, viral load, and genome fraction yield in patients with Middle East respiratory syndrome," J Infect Dis. Vol. 210, no. 10, pp. 1590-1594, 2014.

[41] US Centers for Disease Control and Prevention. December 2020. Interim guidelines for collecting, handling, and testing clinical specimens from persons under investigation (PUIs) for coronavirus disease 2019 (COVID-19) (Online). Available: https://www.cdc. gov/coronavirus/2019-nCoV/lab/guidelinesclinicalspecimens.html.

[42] P. Sarma, H. Kaur, H. Kumar, et al., "Virological and clinical cure in Covid-19 patients treated with hydroxychloroquine: a systematic review and meta-analysis," J Med Virol., vol. 92, pp. 776-785, 2020.

[43] M. Marmor, U. Kellner, T.Y. Lai, et al., "Recommendations on screening for chloroquine and hydroxy-chloroquine retinopathy (2016 revision)," Ophthalmology, vol. 123, no. 6, pp. 1386-1394, 2016.

[44] The Center of Evidence-Based Medicine. April 2020. Lopinavir/ritonavir: a rapid review of effectiveness in COVID-19 (Online). Available: https://www.cebm.net/covid-19/lopinavirritonavir-a-rapid-review-of-the-evidence-for-effectiveness-in-treatingcovid/.

[45] D.M. Varu, M.K. Rhee, E.K. Akpek, et al., "Conjunctivitis Preferred Practice Pattern," Ophthalmology, pp. 94-169, 2019.

[46] R. Shetty, A. Ghosh, S.G. Honavar, et al., "Therapeutic opportunities to manage COVID-19/SARS-CoV-2 infection: present and future," Indian J Ophthalmol., vol.68, pp. 693-702, 2020.

[47] M. Day, "Covid-19: Ibuprofen should not be used for managing symptoms, say doctors and scientists," BMJ, pp. 368:m1086, 2020.

[48] A. Bhavsar, S. Bhavsar, S. Jain, "Evaluation of the effects of chloroquine phosphate eye drops in patients with dry eye syndrome," Int J Biomed Adv Res, vol. 2, pp. 198-214, 2011.

[49] V. Navel, F. Chiambaretta, F. Dutheil, "Haemorrhagic conjunctivitis with pseudomembranous related to SARS-CoV-2," American Journal of Ophthalmology Case Reports, vol. 19, pp. 1-4, 2020. 